\title{
STAT3 activity regulates sensitivity to tumor necrosis factor-related apoptosis-inducing ligand-induced apoptosis in cervical cancer cells
}

\author{
HIROE NAKAMURA* , AYUMI TAGUCHI ${ }^{*}$, KEI KAWANA, AKIRA KAWATA, MITSUYO YOSHIDA, \\ ASAHA FUJIMOTO, JURI OGISHIMA, MASAKAZU SATO, TOMOKO INOUE, HARUKA NISHIDA, \\ HITOMI FURUYA, KENSUKE TOMIO, SATOKO EGUCHI, MAYUYO MORI-UCHINO, \\ AKI YAMASHITA, KATSUYUKI ADACHI, TAKAHIDE ARIMOTO, OSAMU WADA-HIRAIKE, \\ KATSUTOSHI ODA, TAKESHI NAGAMATSU, YUTAKA OSUGA and TOMOYUKI FUJII \\ Department of Obstetrics and Gynecology, Graduate School of Medicine, \\ The University of Tokyo, Bunkyo-ku, Tokyo 113-8655, Japan
}

Received July 3, 2016; Accepted August 16, 2016

DOI: $10.3892 /$ ijo.2016.3681

\begin{abstract}
In cervical cancer, p53-induced apoptosis is abrogated by human papilloma virus (HPV)-derived oncoprotein E6. Although tumor necrosis factor-related apoptosis-inducing ligand (TRAIL) provides tumor-specific apoptosis in various cancers, including cervical cancer, the sensitivity differs depending on the cell lines. Signal transducer and activator of transcription 3 (STAT3) is a hub molecule that shifts the cellular fate to apoptosis or survival in response to cellular stresses. However, the contribution of STAT3 activity to TRAILinduced apoptosis in cervical cancer remains unknown. We examined the TRAIL sensitivity in cervical cancer cells, using TRAIL-resistant ( $\mathrm{SiHa}$ ) and -sensitive (CaSki) cervical cancer cell lines and focused on STAT3 function involving the apoptotic pathway. STAT3 was inactivated by TRAIL stimulation in the CaSki cell line, but not in the SiHa cell line. We then inhibited STAT3 expression in the SiHa cell line using siRNA against STAT3 and suppressed STAT3 activity using a STAT3 inhibitor; both these treatments sensitized TRAIL-induced apoptosis in the SiHa cell line. Furthermore, the SiHa cells were exposed to tunicamycin (TM), an endoplasmic reticulum (ER) stress inducer that inactivates STAT3, with or without
\end{abstract}

Correspondence to: Dr Kei Kawana, Department of Obstetrics and Gynecology, Graduate School of Medicine, The University of Tokyo, 7-3-1 Hongo, Bunkyo-ku, Tokyo 113-8655, Japan

E-mail: kkawana-tky@umin.org

${ }^{*}$ Contributed equally

Abbreviations: TRAIL, tumor necrosis factor-related apoptosis inducing ligand; STAT3, signal transducer and activator of transcription 3

Key words: TRAIL, STAT3, cervical cancer, apoptosis, ER stress
TRAIL. Accompanied by STAT3 inactivation, TM pretreatment significantly enhanced TRAIL-induced apoptosis. We therefore concluded that TRAIL-induced apoptosis was regulated by STAT3 in response to TRAIL stimulation. Our results also suggest that STAT3 inhibition increases the sensitivity of malignancies, particularly HPV-related cancer, to TRAILbased therapy.

\section{Introduction}

Cervical cancer is the most common carcinoma caused by oncogenic human papillomavirus (HPV). Globally, it accounted for an estimated 528,000 new cancer cases worldwide and 266,000 deaths in 2012 (1) even though HPV vaccination programs have been implemented worldwide. Conventional therapy for advanced cervical cancer is radiotherapy, chemotherapy, or both. However, some populations with chemo-resistance have poor prognoses. Alternative therapeutics including molecule-targeting agents have not yet been developed for cervical cancer.

Tumor necrosis factor-related apoptosis-inducing ligand (TRAIL) is a member of the TNF superfamily and has received attention for its role as an antitumor agent that has few cytotoxic effects on normal cells (2-4). Two major pathways exist downstream of TRAIL signaling: pro-apoptosis and pro-survival signaling (5). The apoptosis pathway directly induces the TRAIL-R1/2-DISC (death-inducing signaling complex)-induced activation of caspase- 8 or triggers apoptosis indirectly via a mitochondrial pathway. On the other hand, the pro-survival pathway induces the activation of $\mathrm{NF}-\kappa \mathrm{B}$ or JNK/ p38 signaling and increases the expression of anti-apoptotic or proliferation stimulatory proteins (5). The fate of TRAIL signaling is controlled by the balance between pro-apoptotic and pro-survival signaling, which varies among tumor cell types.

In cervical cancer cells, p53-induced apoptosis is abrogated through the degradation of p53 by the HPV E6 oncoprotein (6) and thus, the other types of apoptosis pathway such as 
TRAIL-induced apoptosis pathway appear to play a major role in cell programmed death. Overcoming TRAIL resistance has a potential to improve outcome in treating cervical cancer. A previous study reported that sensitivity to TRAIL-induced apoptosis differed among cell lines and that the proteasome inhibitor MG132 sensitized TRAIL-induced apoptosis in cervical cancer cells by upregulating death receptor (DR) 4/5 and inactivating X-linked inhibitor of apoptosis (XIAP) (7). However, the biological mechanisms preventing TRAILinduced apoptosis in several cell lines have not yet been elucidated in detail.

Signal transducer and activator of transcription 3 (STAT3) is activated by tyrosine phosphorylation in response to various cytokine stimuli (8-10). STAT3 is induced by various types of cellular stresses such as hypoxia, reperfusion, and ultraviolet (UV) (11), which often occur in the tumor microenvironment (TME). STAT3 and its downstream p38 mitogen-activated protein kinase (MAPK) signaling pathway are well-known regulators of apoptosis or survival of damaged cells (11-13). The constitutive activation of STAT3 has been reported in various types of malignancies, including cervical cancer (14-18). Notably, the inhibition of STAT3 has been shown to shift some apoptosis-resistant cells to TRAIL-induced apoptosis $(19,20)$. Numerous reports have demonstrated that several components with the potency to suppress STAT3 activation such as histone deacetylase (HDAC) inhibitors (21-25), resveratrol (26-30), and curcumin (31-35) enhanced TRAIL-induced apoptosis in TRAIL-resistant cells.

Due to the importance of the STAT3 pathway inhibition and TRAIL-induced apoptosis especially in cervical cancer therapy, we attempted to elucidate the mechanisms responsible for TRAIL-induced apoptosis with a focus on STAT3 activity.

\section{Materials and methods}

Antibodies and reagents. The following antibodies were used at the dilutions indicated. In western blotting, mouse antihuman $\alpha$-Tubulin sc-8035 (1:500) purchased from Santa Cruz Biotechnology (TX, USA), mouse anti-human total STAT3 (124H6) CS\#9139 (1:1,000), rabbit anti-human phosphoSTAT3 (Tyr705) (D3A7) CS\#9145 (1:1,000), purchased from Cell Signaling Technologies (MA, USA). The STAT3 inhibitor S3I-201 was purchased from Santa Cruz Biotechnology. Recombinant human TRAIL was purchased from R\&D Systems (Minnesota, MN, USA).

Cell cultures. The cervical cancer cell lines SiHa and CaSki were maintained in Dulbecco's modified Eagle's medium (Wako, Osaka Japan) with 10\% fetal bovine serum (FBS, Life Technologies, CA, USA) and antibiotics (antibiotic-antimycotic mixed stock solution, Nacalai Tesque, Kyoto, Japan). Cells were grown in a humidified tissue culture incubator at $37^{\circ} \mathrm{C}$ in $5 \% \mathrm{CO}_{2}$.

Cell proliferation assay. Cell proliferation assays were performed to analyze the effects of TRAIL, the STAT3 inhibitor S3I-201, tunicamycin on cell proliferation. Five thousand cells were seeded on 96-well plates. Cell Counting Kit-8 (CCK-8) with the tetrazolium salt WST-8 [2-(2-methoxy-4-
nitrophenyl)-3-(4-nitrophenyl)-5-(2,4-disulfophenyl)-2H-tetrazolium, monosodium salt] (Dojindo, Osaka, Japan) was used and quantified by monitoring changes in absorbance at $450 \mathrm{~nm}$, which were normalized relative to the absorbance of control cells. The lengths of the treatments are described with each result.

Detection of apoptosis by staining with Annexin V-FITC. Cells $\left(4 \times 10^{5}\right)$ were cultured in 6 -well plates for $24 \mathrm{~h}$ before being treated. SiHa was also pretreated with S3I-201 or tunicamycin for $24 \mathrm{~h}$ with an additional 15-18 $\mathrm{h}$ of the TRAIL treatment. Cells were trypsinized, washed with PBS, and then analyzed after double staining with an Annexin V Apoptosis Detection kit (Abcam, MA, USA). The apoptotic cell population was assessed using flow cytometry.

Immunoblotting. Total cellular extracts were prepared by lysing cells from dishes in lysis buffer (\#9803, Cell Signaling Technologies) containing protease inhibitor cocktail (Nacalai Tesque) and phosphatase inhibitor cocktail (Roche, Mannheim, Germany) on ice for $5 \mathrm{~min}$, and then sonicated briefly. Cells were centrifuged at $14,000 \mathrm{rpm}$ at $4^{\circ} \mathrm{C}$ for $10 \mathrm{~min}$. The supernatant was used in subsequent analyses. In SDS-PAGE, $20 \mu \mathrm{g}$ of the protein lysate with $6 \mathrm{X}$ sample buffer (Nacalai Tesque) was loaded into each well. In immunoblotting, $0.45-\mu \mathrm{m}$ PVDF membranes (Merck Millipore, Darmstadt, Germany) were used. The membranes were blocked in 5\% milk/TBS-T (TBS containing $0.1 \%$ Tween-20) at room temperature for $1 \mathrm{~h}$ followed by an incubation with the primary antibodies diluted in 5\% milk/TBS-T or 5\% BSA/TBS-T for the appropriate time period indicated in the manufacturer's instructions. After several washes with TBS-T, the membranes were incubated with secondary antibodies conjugated with HRP in 5\% milk/ TBS-T at room temperature for $1 \mathrm{~h}$. Blots were developed using Immobilon Western Chemiluminescent HRP substrate (Merck Millipore) according to the manufacturer's instructions.

Transfections. Small interfering RNA transfections were performed using Stealth RNAi against STAT3 (HSS186130, HSS186131, and HSS110279) and non-targeting siRNA (Stealth RNAi ${ }^{\mathrm{TM}}$ siRNA Negative Control, Med GC, Life Technologies) as a control. When cells were $60-70 \%$ confluent, transfections were performed using Lipofectamine RNAiMAX (Life Technologies), Opti-MEM reduced serum medium (Life Technologies), and a final concentration of $20 \mathrm{nmol} / \mathrm{l}$ of siRNAs, according to the manufacturer's instructions. After $5 \mathrm{~h}$ of incubation, the transfection medium was changed to normal culture medium without antibiotics. Cells were incubated for $48 \mathrm{~h}$ and then analyzed for each experiment. The transfection sequence was repeated at least 3 times.

$R T$-quantitative PCR. Total RNA was extracted from cells using Blood/Cultured Cell Total RNA Mini kit (Favorgen, Ping Tung, Taiwan), followed by reverse transcription. cDNA was amplified for 40 cycles in a Light Cycler 480 (Roshe). Expression of CHOP was normalized by GAPDH mRNA as an internal standard, and spliced XBP1 (sXBP1) was normalized by total XBP1 (tXBP1) calculated by the $\Delta \Delta \mathrm{Cq}$ method. The primer pairs were as follows: human total XBP1 (tXBP1) 5'-GGCATCCTT 
A

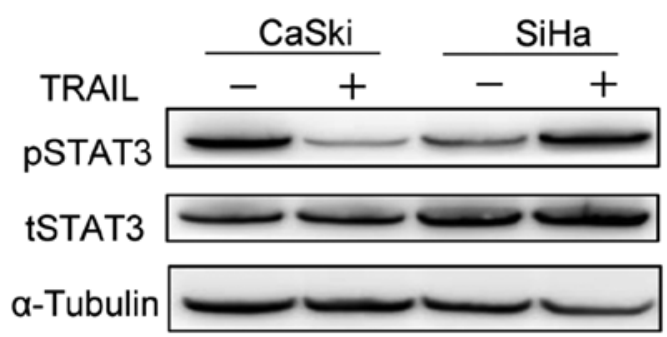

B

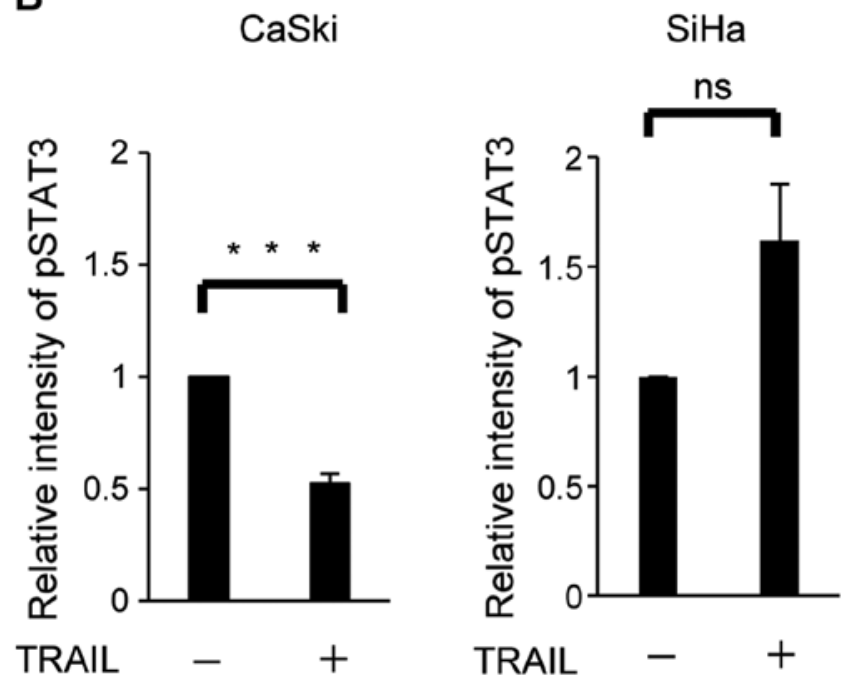

Figure 1. STAT3 activity is suppressed by TRAIL in CaSki but not in SiHa. Protein levels of phospho-STAT3 (pSTAT3) and total STAT3 (tSTAT3) after a TRAIL treatment were examined. (A) CaSki and SiHa were treated with TRAIL (100 ng/ml) for $4 \mathrm{~h}$ and lysed in cell lysis buffer. They were then analyzed using western blotting. Data are representative of six independent experiments. (B) Relative intensities of the blots normalized to $\alpha$-Tubulin. Data are the mean $\left( \pm\right.$ SEM) of six independent experiments. ${ }^{* * *} \mathrm{P}<0.0005$; NS, not significant.

GGCTTGCCTCCA-3', and 5'-GCCCCCTCAGCAGGTGT TCC-3', human spliced XBP1 (sXBP1) 5'-CGCTTGGGGAT GGATGCCCTG-3' and 5'-CCTGCACCTGCTGCGGACT-3', human CHOP 5'-GGAGCATCAGTCCCCCACTT-3' and 5'-TGTGGGATTGAGGGTCACATC-3', and human GAPDH 5'-GAAAGGTGAAGGTCGGAGTC-3' and 5'-GAAGATGG TGATGGGATTTC-3'.

Statistical analysis. Data are presented as means \pm standard error of the mean (SEM). Statistical analyses were carried out using Student's t-test or Dunett's analysis using JMP software. A value of $\mathrm{P}<0.05$ was considered significant. In the figure legends, asterisks indicate comparisons with significant difference $(\mathrm{P}<0.05)$.

\section{Results}

STAT3 activation is suppressed in CaSki by the TRAIL stimulation. Hougardy et al previously reported that HPV16positive cervical cell line CaSki is the most sensitive cell line to TRAIL-induced apoptosis while HPV16-positive SiHa is the most resistant one among cervical cancer cell lines that were tested (7).

In order to investigate the cause of the difference in TRAIL sensitivity, we first performed a comprehensive analysis on apoptotic signaling in both cell types under physiological conditions using an apoptosis array. However, no marked differences were observed in the expression levels of apoptosis-related molecules including pro-apoptotic protein survivin or TRAIL receptors: DR4 and DR5 (data not shown).

STAT3 has been reported to be involved in apoptosis resistance in various cancers (36-38). We investigated the involvement of STAT3 in TRAIL sensitivity between CaSki and $\mathrm{SiHa}$ (Fig. 1). Western blotting revealed that the expression levels of total STAT3 (tSTAT3) were similar in both cell types regardless of the TRAIL stimulation. The phosphorylation of STAT3 (pSTAT3) was suppressed in CaSki by TRAIL, but tend to be upregulated or remained at the same level in SiHa (Fig. 1, upper panels). Six independent experiments revealed that the protein level of pSTAT3 decreased significantly $(\mathrm{P}=0.0003)$ by TRAIL in CaSki, but not in SiHa $(\mathrm{P}=0.0957)$ (Fig. 1, lower panels). These results indicated that the modulation of STAT3 activity by TRAIL might be involved in the different responses to the TRAIL stimulation.

siSTAT3 enhances TRAIL-induced apoptosis. In order to examine the role of STAT3 in resistance to TRAIL-induced apoptosis, optimized siRNA for STAT3 (siSTAT3) or control siRNA was transduced into SiHa (Fig. 2). Total STAT3 (tSTAT3) as well as phosphorylation of STAT3 (pSTAT3) expression were sufficiently knocked-down at the protein level by siSTAT3 in SiHa (Fig. 2A). STAT3 knockdown (siSTAT3) and control cells were exposed to TRAIL and then assessed for apoptosis. Although the TRAIL stimulation induced apoptosis in control and siSTAT3 cells, the extent of apoptosis observed was markedly higher in siSTAT3 cells $[21.2( \pm 2.6) \%]$ than in control cells $[8.7( \pm 0.44) \%]$ (Fig. 2B). The increase induced in apoptosis by the knockdown of STAT3 did not occur in cells not exposed to TRAIL. These results indicated that the knockdown of STAT3 enhanced sensitivity to TRAIL-induced apoptosis in $\mathrm{SiHa}$.

Next, we investigated the effect of STAT3 suppression on cisplatin (CDDP)-induced apoptosis in the SiHa cells, as CDDP is one of the standard chemotherapeutics for advanced or recurrent cervical cancer (39). Unlike TRAIL-induced apoptosis, STAT3 inhibition did not enhance CDDP-induced apoptosis in the SiHa cells (data not shown).

The STAT3 inhibitor enhances sensitivity to TRAIL-induced apoptosis. As a molecular-targeting agent in a clinical setting, S3I-201 is an attractive STAT3 inhibitor which inhibits STAT3 dimerization, DNA biding and transcriptional activities (40). We hypothesized that S3I-201 may enhance sensitivity to TRAIL-induced apoptosis in cells resistant to TRAIL. Apoptosis was examined in TRAIL-stimulated SiHa cells treated with or without the STAT3 inhibitor S3I-201 (Fig. 3A). Increases of less than 5\% were observed in the proportion of apoptotic cells by the TRAIL stimulation without exposure to S3I-201. TRAIL-stimulated SiHa showed a five-fold increase $[4.3( \pm 0.97) \%$ to $23( \pm 2.1) \%]$ in the proportion of apoptotic cells following exposure to $100 \mu \mathrm{M}$ of S3I-201 (Fig. 3A). 


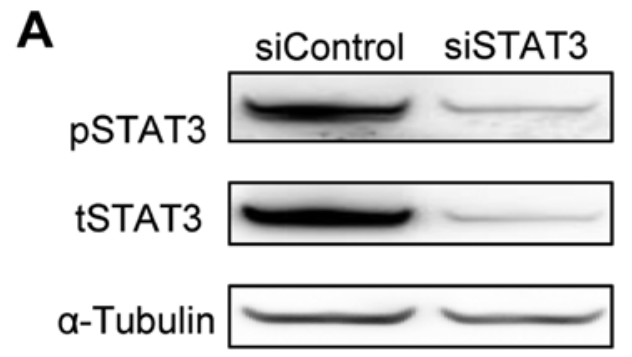

B
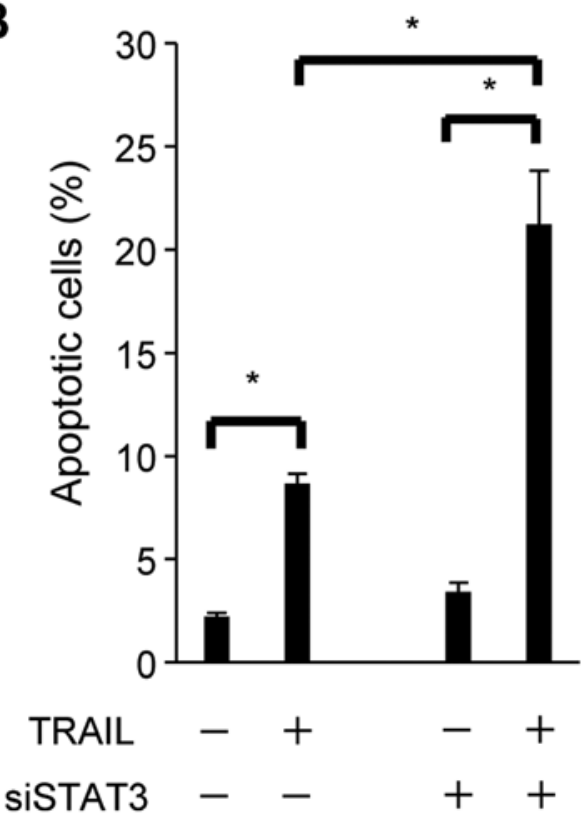

Figure 2. STAT3 knockdown sensitizes TRAIL-induced apoptosis in SiHa. (A) SiHa was transfected with control siRNA (siControl) or STAT3-specific siRNA (siSTAT3) for $48 \mathrm{~h}$ and then analyzed by western blotting to confirm the efficacy of the knockdown. The data are representative of three independent experiments. (B) SiHa was transfected with control siRNA or STAT3-specific siRNA for $48 \mathrm{~h}$, then treated with or without TRAIL $(100 \mathrm{ng} / \mathrm{ml})$ for an additional $15 \mathrm{~h}$. The proportion of apoptotic cells was evaluated using Annexin V-fluorescein isothiocyanate (FITC) and propidium iodide (PI) double staining, followed by an analysis using flow cytometry. The results show the mean of three independent experiments $( \pm$ SEM). The data were analyzed using Student's t-test. ${ }^{*} \mathrm{P}<0.05$.

Furthermore, SiHa cells were exposed to S3I-201 at different doses $(25,50$ and $100 \mu \mathrm{M})$ with or without a sequential $100 \mathrm{ng} /$ $\mathrm{ml}$ of TRAIL stimulation, and cell viability at each dose was blotted on the curve (Fig. 3B). Although S3I-201 alone suppressed cell viability at doses $>50 \mu \mathrm{M}$, the combination of S3I-201 and TRAIL more effectively suppressed cell viability in SiHa. These results indicated that the STAT3 inhibitor, synergistic with TRAIL, enhanced sensitivity to apoptosis in TRAIL-resistant SiHa cell line.

Tunicamycin sensitized TRAIL-induced apoptosis by suppressing STAT3 activation in SiHa. Previous studies demonstrated that several ER stress inducers suppressed STAT3 phosphorylation $(41,42)$. We hypothesized that ER stress inducers may sensitize cells to TRAIL-induced apoptosis through the inactivation of STAT3. The TRAIL-resistant cell line, $\mathrm{SiHa}$, was exposed to different doses of the ER stress inducer, tunicamycin (TM) $(0.01,0.05,0.1,0.5$ and $1.0 \mu \mathrm{M})$ to
A

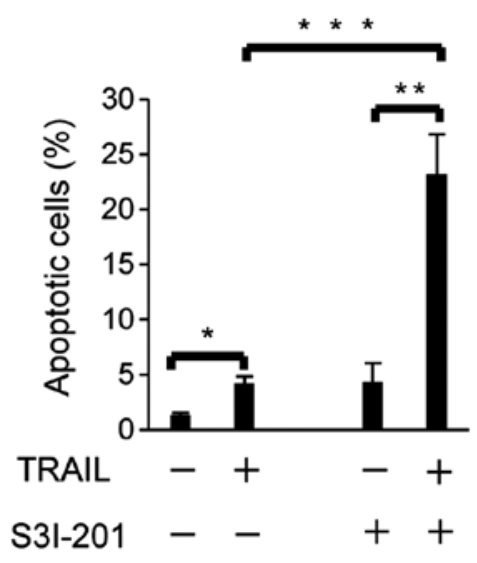

B

$\mathrm{S} 3 \mathrm{I}-201(\mu \mathrm{M}) 0.1$

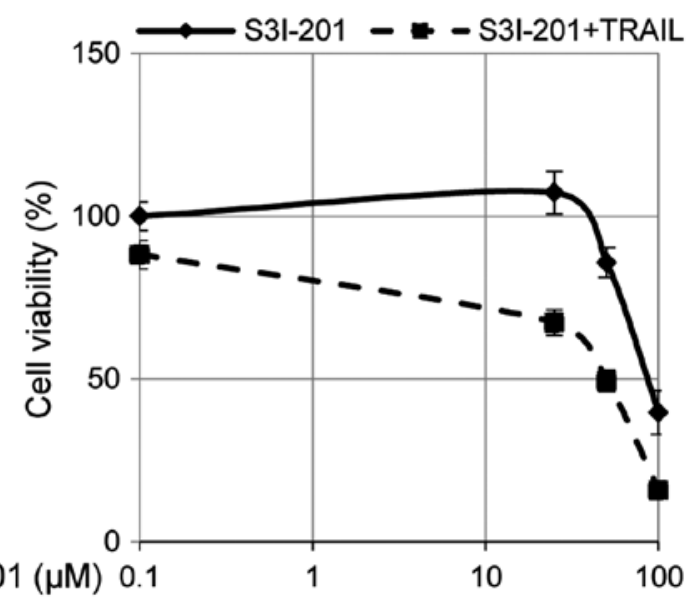

Figure 3. STAT3 inhibitor S3I-201 sensitizes TRAIL-induced apoptosis in SiHa. (A) SiHa was treated with S3I-201 $(100 \mu \mathrm{M})$ for $24 \mathrm{~h}$, then with or without TRAIL (100 ng/ml) for an additional $15 \mathrm{~h}$. The proportion of apoptotic cells was evaluated using Annexin V-fluorescein isothiocyanate (FITC) and propidium iodide (PI) double staining, followed by flow cytometry analysis. The results show the mean of three independent experiments $( \pm$ SEM). The data were analyzed using Student's t-test. ${ }^{*} \mathrm{P}<0.05,{ }^{* *} \mathrm{P}<0.005$. (B) SiHa was treated with S3I-201 at the doses indicated for $24 \mathrm{~h}$, then with or without TRAIL (100 ng/ml) for an additional $15 \mathrm{~h}$. Cell viability was analyzed using a Cell Counting Kit-8 (CKK-8) assay. Each experiment was performed in quadruplicate and the data are the representative of two independent experiments. Data were normalized relative to the value of the control cells. The reported values are the mean $( \pm$ SEM).

examine the phosphorylation of STAT3 and TRAIL-induced apoptosis. We confirmed that TM upregulated C/EBP homologous protein (CHOP) and X-box-binding protein-1 (XBP1) splicing levels in a dose-dependent manner, indicating that TM activated an unfolded protein reaction (UPR): ER stress branches (Fig. 4A). Western blotting for pSTAT3 and tSTAT3 demonstrated that TM successfully suppressed the activation (phosphorylation) of STAT3 in a dose-dependent manner (Fig. 4B).

$\mathrm{SiHa}$ was then exposed to TM at different doses $(0.01$, $0.05,0.1,0.5$ and $1.0 \mu \mathrm{M}$ ) for $24 \mathrm{~h}$ with or without a sequential $100 \mathrm{ng} / \mathrm{ml}$ of TRAIL stimulation, and cell viability was assessed using CCK-8 (Fig. 5A). TM reduced cell viability to less than $50 \%$ at doses greater than $0.1 \mu \mathrm{M}$ in the absence of TRAIL (Fig. 5A). Since TM increased spliced XBP1 and 
A

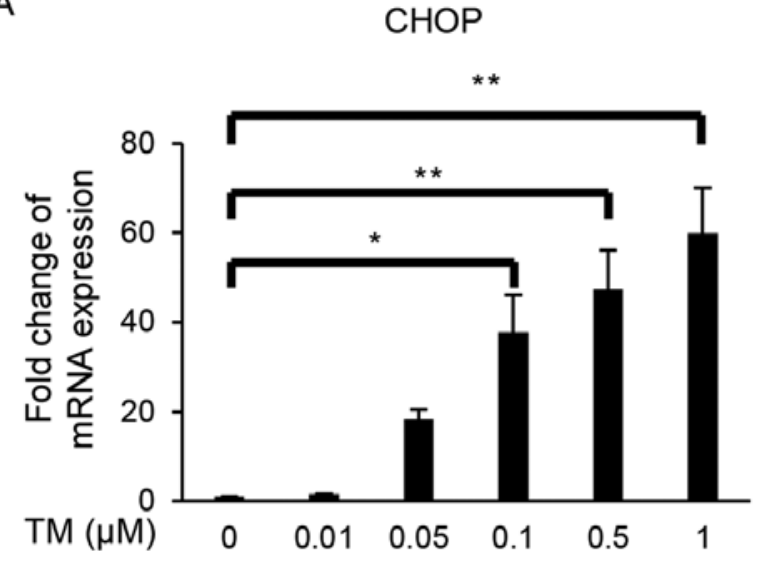

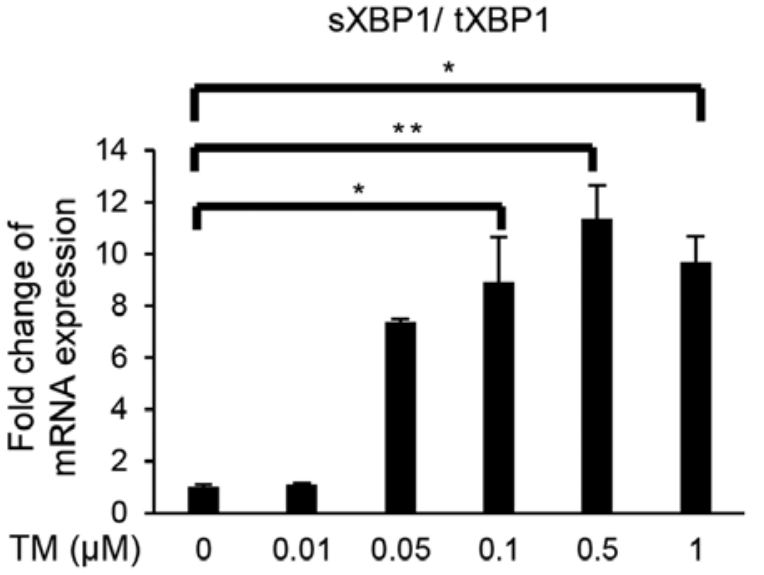

B

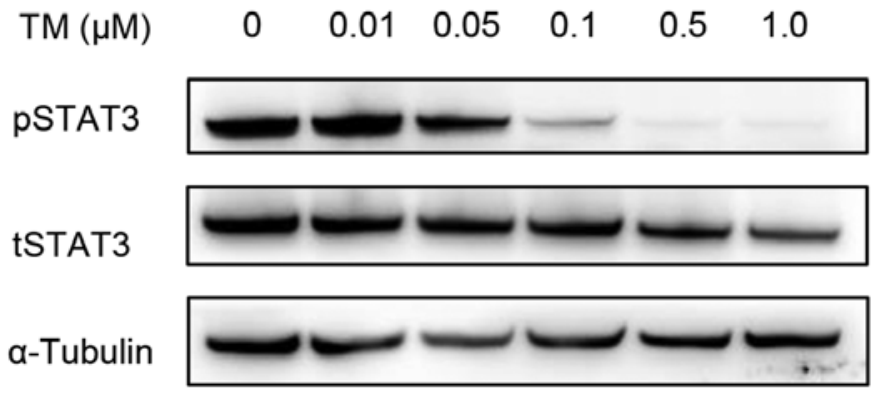

Figure 4. Tunicamycin suppresses STAT3 activity. (A) SiHa was treated with tunicamycin (TM) at the doses indicated for 24 h. Total RNA was reverse transcribed and the mRNA levels of C/EBP homologous protein (CHOP), X-box-binding protein-1 (XBP1) splicing levels (sXBP1), and total XBP1 (tXBP1) were measured by a quantitative reverse transcription polymerase chain reaction. The expression level of CHOP was normalized relative to that of glyceraldehyde 3-phosphate dehydrogenase (GAPDH). The expression level of spliced XBP1 was normalized to that of total XBP1. Data are the mean ( \pm SEM) of three independent experiments and analyzed by Dunett's analysis using JMP software $\left({ }^{*} \mathrm{P}<0.05,{ }^{* *} \mathrm{P}<0.005\right)$. (B) SiHa was treated with TM at the doses indicated for $24 \mathrm{~h}$, lysed in cell lysis buffer, and then STAT3 expression was analyzed by western blotting.

CHOP, which play central roles in ER stress-mediated apoptosis (43) (Fig. 4A), decreases in cell viability appeared to reflect TM-induced ER stress-mediated apoptosis. However, cell viability curves revealed that TM in combination with TRAIL had more suppressive effect on the cell viability compared with TM alone. In the presence of TRAIL, viable cells were barely detected at the same doses of TM (Fig. 5A). We also examined the proportion of apoptotic cells among $\mathrm{SiHa}$ exposed to TRAIL and/or TM at different doses (Fig. 5B). The proportion of apoptotic cells was only $5.9( \pm 0.22) \%$ in $\mathrm{SiHa}$ exposed to TRAIL alone, but increased to $47.1( \pm 3.4) \%$ and $81.0( \pm 0.2) \%$ in SiHa exposed to TRAIL in combination with $\mathrm{TM}$ at 0.05 and $0.1 \mu \mathrm{M}$, respectively. Exposure to TM alone induced apoptosis in 5.8 $( \pm 0.68) \%$ and $12.4( \pm 0.48) \%$ of cells at these doses. Therefore, the combination of TM and TRAIL had a synergistic effect on the induction of apoptosis.

\section{Discussion}

In this study, we showed that STAT3 activation was suppressed by TRAIL in the TRAIL-sensitive cell line CaSki, but not in the TRAIL-resistant SiHa cell line. The inhibition of STAT3 expression using siRNA technology and the suppression of STAT3 activity using a STAT3 inhibitor increased the sensitivity of the SiHa cells to TRAIL-induced apoptosis. Furthermore, an ER stress inducer (TM) also effectively increased their sensitivity to TRAIL-induced apoptosis accompanied by STAT3 inactivation. These results indicated that STAT3 regulated the TRAIL sensitivity in the SiHa cells.

We first examined the differences in the basal expression of apoptosis-related molecules including apoptosis-related receptors. However, no marked differences were observed in the expression levels of apoptosis-related molecules or TRAIL receptors (data not shown). In contrast, western blotting revealed a difference in the phosphorylation of STAT3 with TRAIL stimulation (Fig. 1), whereas other major proapoptotic signaling pathways remained unchanged (data not shown). These results indicate that STAT3 activity might be involved in the difference in the responses of the two cell lines to TRAIL stimulation, which is consistent with previous findings showing that the inhibition of STAT3 signals sensitizes TRAIL-induced apoptosis $(41,42)$. Previous studies reported that several components that have the potency to suppress STAT3 shift the fate of some apoptosis-resistant malignant cells to TRAIL-induced apoptosis. In these studies, a JAK2 inhibitor, histone deacetylase (HDAC) inhibitor, resveratrol, and curcumin were used as inducers of TRAIL-induced apoptosis (21-35). Taken together, these data suggested that STAT3 might be the central molecule in the resistance of the SiHa cells to TRAIL. In this study, we further confirmed that both suppression of STAT3 expression using siRNA technology and inhibition of STAT3 activation using the STAT3 


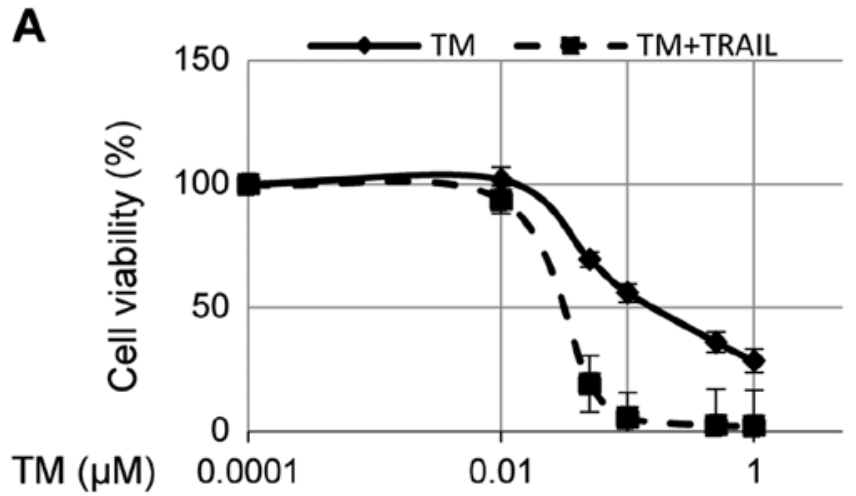

B

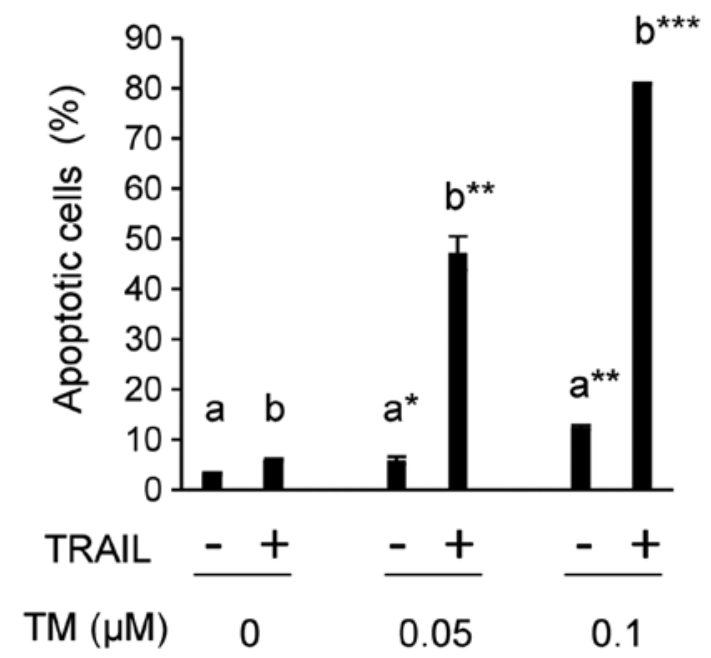

Figure 5. Tunicamycin sensitizes TRAIL-induced apoptosis in SiHa. (A) $\mathrm{SiHa}$ was treated with tunicamycin (TM) at the doses indicated for $24 \mathrm{~h}$, then with or without TRAIL (100 ng/ml) for an additional $15 \mathrm{~h}$. Cell viability was analyzed using a Cell Counting Kit-8 (CKK-8) assay. Data were normalized relative to the value of the control cells. The reported values are the mean of the relative cell viability $( \pm$ SEM). Each experiment was performed in quadruplicate and the data are representative of three independent experiments. (B) SiHa was treated with TM $(0.05$ and $0.1 \mu \mathrm{M})$ for $24 \mathrm{~h}$, then with or without TRAIL $(100 \mathrm{ng} / \mathrm{ml})$ for an additional $15 \mathrm{~h}$. The proportion of apoptotic cells was evaluated using Annexin V-fluorescein isothiocyanate (FITC) and propidium iodide (PI) double staining, followed by flow cytometry analysis. The result is a representative of two independent experiments performed in triplicate. The result shows the mean of apoptosis cell proportion $(\%)( \pm$ SEM). The data were analyzed by Student's t-test using JMP software, regarding ' $a$ ', ' $b$ ' as controls. ${ }^{*} \mathrm{P}<0.05,{ }^{* *} \mathrm{P}<0.005$, ${ }^{* * * *} \mathrm{P}<0.0005$.

inhibitor S3I-201 increased the TRAIL-induced apoptosis in the SiHa cells (Figs. 2 and 3). Oncogenes and p53 are the central regulators of the apoptosis induction in cervical cancer. A previous report demonstrated that knockdown of STAT3 suppressed the expression of viral E6 and E7 oncoproteins as well as upregulated p53 expression (44). Upregulation of p53 might also enhanced the apoptosis-inducing function of the STAT3 knockdown in our model. These results indicated that targeting STAT3 expression or activity combined with TRAIL stimulation could be a strategy for cervical cancer treatment.

ER stress-mediated apoptosis occurs following the disruption of the UPR balance under prolonged ER stress. UPR branches (PERK, IRE1a, and ATF6) are well known to cross-talk with each other and various cellular signaling pathways (45). However, it currently remains unclear whether STAT3 preferentially involves UPR or ER stress-mediated apoptosis. Several studies have shown that some ER stress inducers suppress the phosphorylation of STAT3 $(41,42)$. The results of this study showed that $0.1 \mu \mathrm{M}$ of tunicamycin (TM) upregulated CHOP, which plays a central role in ER stressmediated apoptosis (43), although it induced apoptosis in only $10 \%$ of the treated cells (Fig. $5 \mathrm{~B}$ ). This result indicated that TM activated UPR, but did not induce ER stress-mediated apoptosis at this dose. In contrast, the combination of TRAIL and $0.1 \mu \mathrm{M}$ of TM induced apoptosis in $80 \%$ of the treated cells (Fig. 5B), which suggested that the combination of TRAIL and TM had a synergistic effect on the induction of apoptosis. Although TM is known to itself upregulate DR4 or DR5 and sensitize cells to TRAIL-induced apoptosis (46-49), only a slight increase in DR5 mRNA level in the SiHa cells was observed after TM treatment in our experiments (data not shown). The inactivation of STAT3 by TM may be one of the key mechanisms to increase the sensitivity of SiHa cells to TRAIL-induced apoptosis, which possibly resulted in the synergistic effect observed.

In addition to the TRAIL-induced apoptosis pathway, we also investigated the effect of STAT3 suppression on the CDDP-induced apoptosis pathway. Although previous reports demonstrated that the inhibition of STAT3 enhanced the CDDP-induced apoptosis in various cancers $(50,51)$, in our model, the inhibition of STAT3 did not enhance the CDDP-induced apoptosis. In the CDDP-induced apoptosis, DNA damage is induced by intra-strand or inter-strand crosslink between two adjacent $G$ residues (52). This DNA damage induces p53-dependent apoptosis. However, HPV-positive cervical cancer lacks p53 expression and the DNA damageinduced apoptosis pathway is considered to be different from other types of cancer. The result suggested that STAT3 inhibition could be an effective therapeutic modality for cervical cancer when combined with TRAIL rather than with CDDP.

In conclusion, in this study, we showed that different TRAIL sensitivity among cell lines might be regulated by STAT3 activity and that the inactivation of STAT3 enhances the sensitivity of the cells to TRAIL-induced apoptosis, even in a TRAIL-resistant cancer cell line. Our results suggest the potential of STAT3 inhibition in combination with TRAILbased therapy for cervical cancer.

\section{Acknowledgements}

We would like to thank Dr Terufumi Yokoyama for expert advice on experimental methodologies. We thank Editage (www.editage.com) for English language editing.

\section{References}

1. Ferlay JSI, Ervik M, Dikshit R, Eser S, Mathers C, Rebelo M, Parkin DM, Forman D and Bray F: Incidence/mortality data. GLOBOCAN 2012 1.0, 2012. www.iarc.fr.

2. Pitti RM, Marsters SA, Ruppert S, Donahue CJ, Moore A and Ashkenazi A: Induction of apoptosis by Apo-2 ligand, a new member of the tumor necrosis factor cytokine family. J Biol Chem 271: 12687-12690, 1996.

3. Zhang L and Fang B: Mechanisms of resistance to TRAILinduced apoptosis in cancer. Cancer Gene Ther 12: 228-237, 2005. 
4. Wiley SR, Schooley K, Smolak PJ, Din WS, Huang CP, Nicholl JK, Sutherland GR, Smith TD, Rauch C, Smith CA, et al: Identification and characterization of a new member of the TNF family that induces apoptosis. Immunity 3: 673-682, 1995.

5. Kruyt FA: TRAIL and cancer therapy. Cancer Lett 263: 14-25, 2008.

6. Moody CA and Laimins LA: Human papillomavirus oncoproteins: Pathways to transformation. Nat Rev Cancer 10: 550-560, 2010.

7. Hougardy BM, Maduro JH, van der Zee AG, de Groot DJ, van den Heuvel FA, de Vries EG and de Jong S: Proteasome inhibitor MG132 sensitizes HPV-positive human cervical cancer cells to rhTRAIL-induced apoptosis. Int J Cancer 118: 1892-1900, 2006.

8. Yu H, Lee H, Herrmann A, Buettner R and Jove R: Revisiting STAT3 signalling in cancer: New and unexpected biological functions. Nat Rev Cancer 14: 736-746, 2014.

9. Akira S: Roles of STAT3 defined by tissue-specific gene targeting. Oncogene 19: 2607-2611, 2000.

10. Levy DE and Lee CK: What does Stat3 do? J Clin Invest 109 1143-1148, 2002.

11. Dudley AC, Thomas D, Best J and Jenkins A: The STATs in cell stress-type responses. Cell Commun Signal 2: 8, 2004.

12. Wada T and Penninger JM: Mitogen-activated protein kinases in apoptosis regulation. Oncogene 23: 2838-2849, 2004.

13. Ramírez de Arellano A, Lopez-Pulido EI, Martínez-Neri PA, Estrada Chávez C, González Lucano R, Fafutis-Morris M, Aguilar-Lemarroy A, Muñoz-Valle JF and Pereira-Suárez AL: STAT3 activation is required for the antiapoptotic effects of prolactin in cervical cancer cells. Cancer Cell Int 15: 83, 2015.

14. Banerjee $\mathrm{K}$ and Resat H: Constitutive activation of STAT3 in breast cancer cells: A review. Int J Cancer 138: 2570-2578, 2016.

15. Benekli M, Xia Z, Donohue KA, Ford LA, Pixley LA, Baer MR, Baumann $\mathrm{H}$ and Wetzler M: Constitutive activity of signal transducer and activator of transcription 3 protein in acute myeloid leukemia blasts is associated with short disease-free survival. Blood 99: 252-257, 2002

16. Takemoto S, Ushijima K, Kawano K, Yamaguchi T, Terada A Fujiyoshi N, Nishio S, Tsuda N, Ijichi M, Kakuma T, et al: Expression of activated signal transducer and activator of transcription-3 predicts poor prognosis in cervical squamous-cell carcinoma. Br J Cancer 101: 967-972, 2009.

17. Schoppmann SF, Jesch B, Friedrich J, Jomrich G, Maroske F and Birner P: Phosphorylation of signal transducer and activator of transcription 3 (STAT3) correlates with Her-2 status, carbonic anhydrase 9 expression and prognosis in esophageal cancer. Clin Exp Metastasis 29: 615-624, 2012.

18. Mali SB: Review of STAT3 (Signal Transducers and Activators of Transcription) in head and neck cancer. Oral Oncol 51: 565-569, 2015.

19. Kusaba M, Nakao K, Goto T, Nishimura D, Kawashimo H, Shibata H, Motoyoshi Y, Taura N, Ichikawa T, Hamasaki K, et al Abrogation of constitutive STAT3 activity sensitizes human hepatoma cells to TRAIL-mediated apoptosis. J Hepatol 47 546-555, 2007

20. Lanuti P, Bertagnolo V, Pierdomenico L, Bascelli A, Santavenere E, Alinari L, Capitani S, Miscia S and Marchisio M: Enhancement of TRAIL cytotoxicity by AG-490 in human ALL cells is characterized by downregulation of cIAP-1 and cIAP-2 through inhibition of Jak2/Stat3. Cell Res 19: 1079-1089, 2009.

21. Singh TR, Shankar S and Srivastava RK: HDAC inhibitors enhance the apoptosis-inducing potential of TRAIL in breast carcinoma. Oncogene 24: 4609-4623, 2005.

22. Fulda S: Histone deacetylase (HDAC) inhibitors and regulation of TRAIL-induced apoptosis. Exp Cell Res 318: 1208-1212, 2012.

23. Earel JK Jr, VanOosten RL and Griffith TS: Histone deacetylase inhibitors modulate the sensitivity of tumor necrosis factorrelated apoptosis-inducing ligand-resistant bladder tumor cells Cancer Res 66: 499-507, 2006.

24. Fandy TE, Shankar S, Ross DD, Sausville E and Srivastava RK: Interactive effects of HDAC inhibitors and TRAIL on apoptosis are associated with changes in mitochondrial functions and expressions of cell cycle regulatory genes in multiple myeloma. Neoplasia 7: 646-657, 2005.

25. Gupta M, Han JJ, Stenson M, Wellik L and Witzig TE: Regulation of STAT3 by histone deacetylase-3 in diffuse large B-cell lymphoma: Implications for therapy. Leukemia 26: 1356-1364, 2012 .
26. Ganapathy S, Chen Q, Singh KP, Shankar S and Srivastava RK Resveratrol enhances antitumor activity of TRAIL in prostate cancer xenografts through activation of FOXO transcription factor. PLoS One 5: e15627, 2010.

27. Taguchi A, Koga K, Kawana K, Makabe T, Sue F, Miyashita M, Yoshida M, Urata Y, Izumi G, Tkamura M, et al: Resveratrol enhances apoptosis in endometriotic stromal cells. Am J Reprod Immunol 75: 486-492, 2016.

28. Quoc Trung L, Espinoza JL, Takami A and Nakao S: Resveratrol induces cell cycle arrest and apoptosis in malignant NK cells via JAK2/STAT3 pathway inhibition. PLoS One 8: e55183, 2013.

29. Kotha A, Sekharam M, Cilenti L, Siddiquee K, Khaled A, Zervos AS, Carter B, Turkson J and Jove R: Resveratrol inhibits $\mathrm{Src}$ and Stat 3 signaling and induces the apoptosis of malignant cells containing activated Stat 3 protein. Mol Cancer Ther 5: 621-629, 2006

30. Tameda M, Sugimoto K, Shiraki K, Inagaki Y, Ogura S, Kasai C, Yoneda M, Okamoto R, Yamamoto N, Takei Y, et al: Resveratrol sensitizes HepG2 cells to TRAIL-induced apoptosis. Anticancer Drugs 25: 1028-1034, 2014

31. Bharti AC, Donato N and Aggarwal BB: Curcumin (diferuloylmethane) inhibits constitutive and IL-6-inducible STAT3 phosphorylation in human multiple myeloma cells. J Immunol 171: 3863-3871, 2003.

32. Alexandrow MG, Song LJ, Altiok S, Gray J, Haura EB and Kumar NB: Curcumin: A novel Stat3 pathway inhibitor for chemoprevention of lung cancer. Eur J Cancer Prev 21: 407-412, 2012.

33. Shankar S, Chen Q, Sarva K, Siddiqui I and Srivastava RK: Curcumin enhances the apoptosis-inducing potential of TRAIL in prostate cancer cells: Molecular mechanisms of apoptosis, migration and angiogenesis. J Mol Signal 2: 10, 2007.

34. Park S, Cho DH, Andera L, Suh N and Kim I: Curcumin enhances TRAIL-induced apoptosis of breast cancer cells by regulating apoptosis-related proteins. Mol Cell Biochem 383: 39-48, 2013.

35. Glienke W, Maute L, Wicht J and Bergmann L: Curcumin inhibits constitutive STAT3 phosphorylation in human pancreatic cancer cell lines and downregulation of survivin/BIRC5 gene expression. Cancer Invest 28: 166-171, 2010.

36. Yu H, Kortylewski M and Pardoll D: Crosstalk between cancer and immune cells: Role of STAT3 in the tumour microenvironment. Nat Rev Immunol 7: 41-51, 2007.

37. Zhang HF and Lai R: STAT3 in Cancer - Friend or Foe? Cancers (Basel) 6: 1408-1440, 2014.

38. Pensa S, Regis G, Boselli D, Novelli F and Poli V: STAT1 and STAT3 in tumorigenesis: Two sides of the same coin. In: Madame Curie Bioscience Database. Landes Bioscience, Austin, TX, 2009.

39. Pectasides D, Kamposioras K, Papaxoinis G and Pectasides E: Chemotherapy for recurrent cervical cancer. Cancer Treat Rev 34: 603-613, 2008

40. Siddiquee K, Zhang S, Guida WC, Blaskovich MA, Greedy B, Lawrence HR, Yip ML, Jove R, McLaughlin MM, Lawrence NJ, et al: Selective chemical probe inhibitor of Stat3, identified through structure-based virtual screening, induces antitumor activity. Proc Natl Acad Sci USA 104: 7391-7396, 2007.

41. Kimura K, Yamada T, Matsumoto M, Kido Y, Hosooka T, Asahara S, Matsuda T, Ota T, Watanabe H, Sai Y, et al: Endoplasmic reticulum stress inhibits STAT3-dependent suppression of hepatic gluconeogenesis via dephosphorylation and deacetylation. Diabetes 61: 61-73, 2012.

42. Yang Z, Liu Y, Liao J, Gong C, Sun C, Zhou X, Wei X, Zhang T, Gao Q, Ma D, et al: Quercetin induces endoplasmic reticulum stress to enhance cDDP cytotoxicity in ovarian cancer: Involvement of STAT3 signaling. FEBS J 282: 1111-1125, 2015

43. Oyadomari S and Mori M: Roles of CHOP/GADD153 in endoplasmic reticulum stress. Cell Death Differ 11: 381-389, 2004

44. Shukla S, Mahata S, Shishodia G, Pandey A, Tyagi A, Vishnoi K, Basir SF, Das BC and Bharti AC: Functional regulatory role of STAT3 in HPV16-mediated cervical carcinogenesis. PLoS One 8: e67849, 2013

45. Hetz C: The unfolded protein response: Controlling cell fate decisions under ER stress and beyond. Nat Rev Mol Cell Biol 13: 89-102, 2012

46. Jiang CC, Chen LH, Gillespie S, Kiejda KA, Mhaidat N, Wang YF, Thorne R, Zhang XD and Hersey P: Tunicamycin sensitizes human melanoma cells to tumor necrosis factor-related apoptosis-inducing ligand-induced apoptosis by up-regulation of TRAIL-R2 via the unfolded protein response. Cancer Res 67: 5880-5888, 2007. 
47. Jung YH, Lim EJ, Heo J, Kwon TK and Kim YH: Tunicamycin sensitizes human prostate cells to TRAIL-induced apoptosis by upregulation of TRAIL receptors and downregulation of cIAP2. Int J Oncol 40: 1941-1948, 2012.

48. Hasegawa A, Osuga Y, Hirota Y, Hamasaki K, Kodama A Harada M, Tajima T, Takemura Y, Hirata T, Yoshino O, et al: Tunicamycin enhances the apoptosis induced by tumor necrosis factor-related apoptosis-inducing ligand in endometriotic stromal cells. Hum Reprod 24: 408-414, 2009.

49. Shiraishi T, Yoshida T, Nakata S, Horinaka M, Wakada M, Mizutani Y, Miki T and Sakai T: Tunicamycin enhances tumor necrosis factor-related apoptosis-inducing ligand-induced apoptosis in human prostate cancer cells. Cancer Res 65: 6364-6370, 2005.
50. Hu Y, Hong Y, Xu Y, Liu P, Guo DH and Chen Y: Inhibition of the JAK/STAT pathway with ruxolitinib overcomes cisplatin resistance in non-small-cell lung cancer NSCLC. Apoptosis 19: 1627-1636, 2014

51. Selvendiran K, Bratasz A, Kuppusamy ML, Tazi MF, Rivera BK and Kuppusamy P: Hypoxia induces chemoresistance in ovarian cancer cells by activation of signal transducer and activator of transcription 3. Int J Cancer 125: 2198-2204, 2009.

52. Dasari S and Tchounwou PB: Cisplatin in cancer therapy: Molecular mechanisms of action. Eur J Pharmacol 740: 364-378, 2014. 